\title{
A taxonomy and mapping of computer-based critiquing tools.
}

\begin{abstract}
Critics have emerged in recent times as a specific tool feature to support users in computermediated tasks. These computer-supported critics provide proactive guidelines or suggestions for improvement to designs, code, and other digital artifacts. The concept of a critic has been adopted in various domains, including medical, programming, software engineering, design sketching, and others. Critics have been shown to be an effective mechanism for providing feedback to users. We propose a new critic taxonomy based on extensive review of the critic literature. The groups and elements of our critic taxonomy are presented and explained collectively with examples, including the mapping of 13 existing critic tools, predominantly for software engineering and programming education tasks to the taxonomy. We believe this critic taxonomy will assist others in identifying, categorizing, developing, and deploying computer-supported critics in a range of domains.
\end{abstract}

Keyword: Design critics; Critiquing systems; Critic taxonomy; Software tool support; Survey. 